\title{
Metal semiconductor metal photodiodes based on all-epitaxial Ge-on-insulator-on- Si(111), grown by molecular beam epitaxy
}

Pokharia, R., Khiangte, K., Rathore, J., Schmidt, J., Osten, $\mathrm{H}$., et al.

R. S. Pokharia, K. R. Khiangte, J. S. Rathore, J. Schmidt, H. J. Osten, A. Laha, S. Mahapatra, "Metal semiconductor metal photodiodes based on allepitaxial Ge-on-insulator-on- Si(111), grown by molecular beam epitaxy," Proc. SPIE 10914, Optical Components and Materials XVI, 1091417 (27 February 2019); doi: 10.1117/12.2509720

SPIE. Event: SPIE OPTO, 2019, San Francisco, California, United States 


\title{
Metal semiconductor metal photodiodes based on all-epitaxial Ge-on- insulator-on-Si(111), grown by molecular beam epitaxy
}

\author{
R. S. Pokharia ${ }^{1}$, K. R. Khiangte ${ }^{2}$, J. S. Rathore ${ }^{2}$, J. Schmidt ${ }^{3}$, H. J. Osten ${ }^{3}$, A. Laha ${ }^{1}$, S. Mahapatra ${ }^{2}$ \\ ${ }^{1}$ Department of Electrical Engineering, Indian Institute of Technology Bombay, Mumbai, INDIA \\ ${ }^{2}$ Department of Physics, Indian Institute of Technology Bombay, Mumbai, INDIA \\ ${ }^{3}$ Institute of Electronic Materials and Devices, Leibniz Universität Hannover, Schneiderberg 32, D- \\ 30167 Hannover, GERMANY
}

\begin{abstract}
${ }^{1}$ ABSTRACT
We report on the fabrication and characterisation of an all-epitaxial Germanium-on-Insulator (GOI) MetalSemiconductor-Metal (MSM) photodetector. The MSM photodetector is fabricated on a (111)-oriented epitaxial Ge layer, grown on an epitaxial $\mathrm{Gd}_{2} \mathrm{O}_{3} / \mathrm{Si}(111)$ substrate, by molecular beam epitaxy (MBE). The first step is the growth of the 15-nm thick $\mathrm{Gd}_{2} \mathrm{O}_{3}$ epitaxial layer over CMOS-grade silicon, atop which an epitaxial layer of Ge is grown. Near infrared (NIR) MSM photodetectors have been fabricated over the Ge epitaxial layer with an inter-digitated (IDT) contact structure, with an active area of $100 \mu \mathrm{m} \times 124 \mu \mathrm{m}$. For the particular IDT dimensions, the dark current has been measured to be $475 \mu \mathrm{A}$. A responsivity of $\sim 2 \mathrm{~mA} / \mathrm{W}$ is observed at a $-5 \mathrm{~V}$ bias, when excited at $1550 \mathrm{~nm}$.
\end{abstract}

Keywords: Germanium, germanium-on-insulator, NIR photodetector, MSM photodiodes, molecular beam epitaxy, silicon photonics.

\section{INTRODUCTION}

Integration of photonic and electronic elements on a single chip is expected to enhance data transfer rates, while reducing power consumption. Towards this goal, significant progress has been made globally, with the vision of developing electro-photonic integrated circuits (EPIC). III-V compound semiconductor materials, such as gallium arsenide (GaAs) and indium phosphide (InP), continue to dominate the photonics market, due to their excellent light emission and absorption properties [1]. However, their integration with the current CMOS technology is non-trivial, due to crosscontamination issues and high costs involved. On the other hand, the relatively large bandgap of $1.12 \mathrm{eV}$ (indirect) and $3.4 \mathrm{eV}$ (direct) of silicon, limits its use in photo-detection, in the near infrared (NIR) wavelength range. In the recent years, germanium has emerged as a promising group-IV semiconductor for fabrication of high-performance NIR photodetectors. With a direct bandgap of $0.8 \mathrm{eV}$, and an indirect bandgap only $130 \mathrm{meV}$ lower in energy, Ge has a relatively high absorption coefficient in the $1.3 \mu \mathrm{m}$ to $1.55 \mu \mathrm{m}$ wavelength range. Being a group-IV semiconductor, Ge can be monolithically integrated on $\mathrm{Si}$ [2], without any cross-contamination. However, the $4 \%$ lattice mismatch between $\mathrm{Ge}$ and Si poses a major challenge for monolithic integration. Nevertheless, various research groups have successfully demonstrated the growth of device-grade $\mathrm{Ge}$ epitaxial layers on $\mathrm{Si}$, which have also been fabricated into NIR photodetectors [3, 4]. MBE is one of the promising epitaxial growth techniques to achieve monolithic integration of Gebased photonics on the Si platform.

In $\mathrm{Ge} / \mathrm{Si}$ epitaxial layers, photo-excited carriers are generated in the Ge layer, when illuminated with light of suitable wavelength. Although the underlying Si also absorbs the light, carriers are generated deep within the Si substrate. Due to the relatively weak absorption of $\mathrm{Si}$, these carriers take a long time to diffuse to the metal contacts (on top of the $\mathrm{Ge}$ epilayer) and hence, the response of the device dampens. This limits the bandwidth and hence the performance of the

${ }^{1}$ Ravindra Singh Pokharia: ravi.micro@iitb.ac.in

Optical Components and Materials XVI, edited by Shibin Jiang, Michel J. F. Digonnet, Proc. of SPIE Vol. 10914, 1091417 - @ 2019 SPIE · CCC code: 0277-786X/19/\$18 doi: $10.1117 / 12.2509720$ 
photodetectors. To enhance the performance of $\mathrm{Ge} / \mathrm{Si}$ photodetectors, an electrical isolation between $\mathrm{Ge}$ and $\mathrm{Si}$ may be inserted, in so-called GOI engineered substrates [5]. Based on the GOI platform, high performance optical devices have already been demonstrated $[5,6,7]$, but the oxide in all these cases is amorphous in nature, with a wafer bonding step included $[8,9]$.

In this work, we report the growth of epitaxial $\mathrm{Ge}(111)$ on $\mathrm{Gd}_{2} \mathrm{O}_{3} / \mathrm{Si}(111)$ template by molecular beam epitaxy and use this structure for fabrication of GOI-based photodetectors. We demonstrate that the use of an epitaxial oxide layer effectively reduced the dark current of metal-semiconductor-metal (MSM) photodetectors, when compared to similar devices fabricated with $\mathrm{Ge} / \mathrm{Si}$ and Ge. The measured dark current for our GOI photodiodes is $\sim 475 \mu \mathrm{A}$ at bias voltages of $\pm 1 \mathrm{~V}$. A responsivity of nearly $2 \mathrm{~mA} / \mathrm{W}$ was observed for these GOI structures at a bias of $5 \mathrm{~V}$, at $1550 \mathrm{~nm}$. By growing thicker Ge layers on $\mathrm{Gd}_{2} \mathrm{O}_{3} / \mathrm{Si}(111)$ substrates, both lower dark currents and higher responsivity may be achieved.

\section{EXPERIMENTAL DETAILS}

\subsection{GOI growth and characterization}

The Ge epilayer was grown in a RIBER MBE (Compact-12) system at CEN, IIT Bombay on a epi- $\mathrm{Gd}_{2} \mathrm{O}_{3} / \mathrm{Si}(111)$ substrate, grown at the Leibniz University, Hannover. Details of the oxide growth can be found elsewhere [10]. For the Ge growth, the $\mathrm{Gd}_{2} \mathrm{O}_{3} / \mathrm{Si}(111)$ substrate was first baked at $180^{\circ} \mathrm{C}$, for approximately 10 hours, in the fast-entry load-lock chamber of the MBE system. The substrate was then transferred to the ultra-high vacuum (UHV) growth chamber. An initial heating of the substrate to $700^{\circ} \mathrm{C}$ was performed, to obtain a clean and smooth epi- $\mathrm{Gd}_{2} \mathrm{O}_{3}$ surface. Following this heat treatment, approximately 550-nm of Ge was grown at a growth temperature $\left(\mathrm{T}_{\mathrm{G}}\right)$ of $300{ }^{\circ} \mathrm{C}$, with a growth rate of about $1.6 \mathrm{~nm} / \mathrm{min}$. In-situ RHEED (Staib Instruments Inc.) was used to monitor the growth in real time, while HRXRD (Rigaku Smartlab) was used for the structural characterization of the grown epilayer. The high-resolution mode was used for $\omega-2 \theta$ and $\omega$ scans, while $\theta-2 \theta$ scans were performed in a low-resolution mode. A $200 \mathrm{kV}$ high resolution transmission electron microscope (HRTEM) from JEOL was used to record XTEM images along the (1 $1 \overline{1} 0)$ direction.

\subsection{GOI MSM device fabrication and characterization}

First, a 300-nm $\mathrm{SiO}_{2}$-layer was deposited for surface passivation of the epi-Ge surface, by Inductively-Coupled-Plasma Chemical Vapour Deposition (ICPCVD). Thereafter, the oxide surface was patterned for MSM IDT structures by standard photolithography and a combination of dry-etching by fluoride-based plasma and wet etching by buffered hydrofluoric acid (BHF). A thermal evaporation process was used to deposit Au-Sb alloy contacts, and finally a lift-off was carried out to make the finished MSM devices. Post-metallization annealing of the devices was carried out at $375^{\circ} \mathrm{C}$, $450^{\circ} \mathrm{C}, 650^{\circ} \mathrm{C}$ and $850^{\circ} \mathrm{C}$, for $60 \mathrm{~s}$ each. The current voltage measurements were carried out with a Keysight B1500A Semiconductor Device Analyzer, while the photocurrent measurements have been carried out using a Keithley 4200 source meter along with a $1550 \mathrm{~nm}$ laser source from Thorlabs.

\section{RESULTS AND DISCUSSIONS}

\subsection{Germanium films}

While Ge growth on $\mathrm{Gd}_{2} \mathrm{O}_{3} / \mathrm{Si}(111)$ starts in the Volmer-Weber $(\mathrm{V}-\mathrm{W})$ growth mode, the layer-by-layer growth mode is recovered within a duration corresponding to deposition of 11-nm Ge. The in-situ RHEED clearly shows the transition in the growth mode of the epilayer. Figure 1 (a) reflects a clean and flat $\mathrm{Gd}_{2} \mathrm{O}_{3}$ surface just after the heat treatment at 700 ${ }^{0} \mathrm{C}$. After $65 \mathrm{~s}$ of growth, a spotty pattern appears (Figure 1 (b)), indicating island formation in the V-W growth mode. Two types of super-imposing spotty patterns, indicating type-A and type-B stacking of the (111) planes, can be seen in this image [11] [12]. Type-A and type-B stacking differ in the stacking order, in which one type is rotated by $180^{\circ}$ about the surface normal, with respect to the other. Subsequently, the growth of only one of the types takes place, and layer-by- 
layer growth mode recovers, as revealed by Figures 1(c) and 1(d). The streaky pattern of Fig. 1(d) persists till the end of Ge growth. More details on the growth and structural characterization of $\mathrm{Ge}(111) / \mathrm{Gd}_{2} \mathrm{O}_{3}(111) / \mathrm{Si}(111)$ can be found in reference [13].
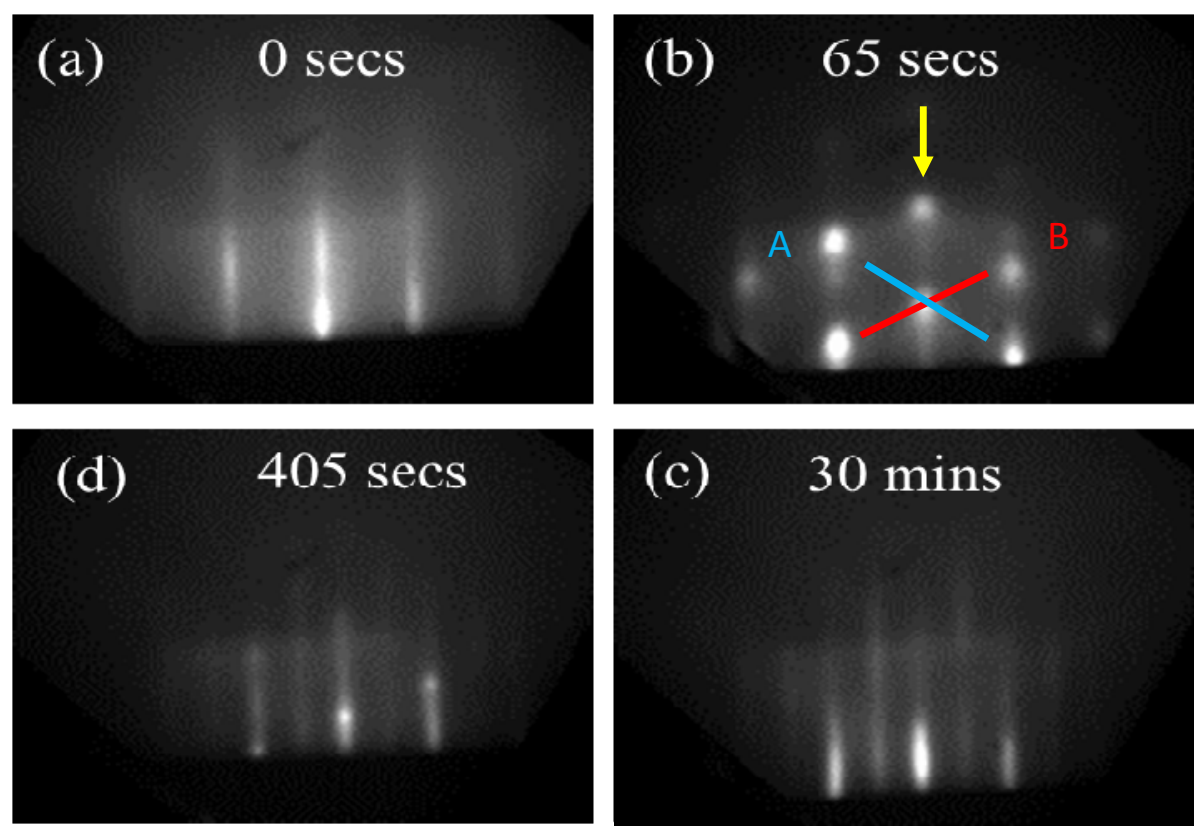

Figure 1. RHEED images recorded at different stages of Ge growth: (a) Image of the $\mathrm{Gd}_{2} \mathrm{O}_{3}$ (111) surface just before the start of Ge growth; (b) $65 \mathrm{~s}$, (c) $405 \mathrm{~s}$ and (d) 35 min after commencement of Ge growth.
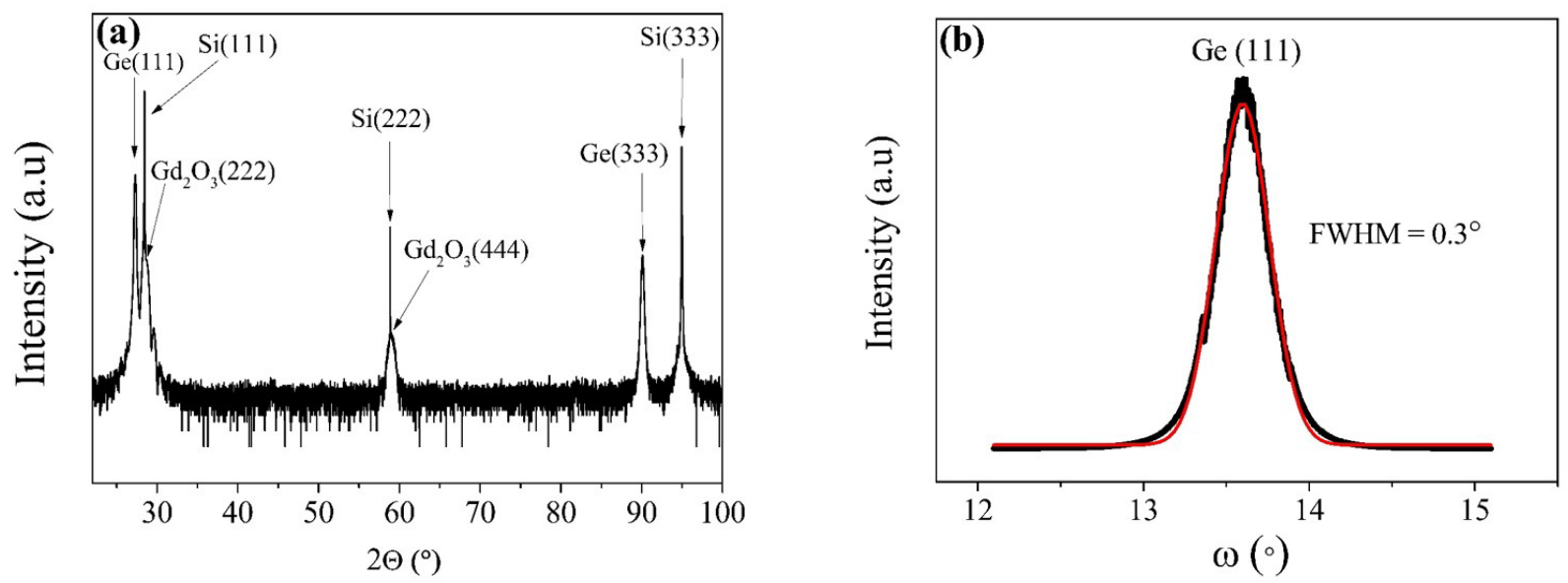

Figure 2. (a) wide $\omega-2 \theta$ scan of the $\mathrm{Ge}(111) / \mathrm{Gd}_{2} \mathrm{O}(111) / \mathrm{Si}(111)$ epilayer and (b) $\omega$-rocking curve diffractogram, recorded around the Ge (111) reflection.

High Resolution X-Rays Diffraction characterization: In $\omega-2 \theta$ XRD-scan, only the (111) and (333) reflections of Ge are visible at $27.30^{\circ}$ and $90.11^{\circ}$, respectively (Figure 2(a)). The rest of the peaks correspond to the $\mathrm{Gd}_{2} \mathrm{O}_{3} / \mathrm{Si}(111)$ substrate. This depicts the formation of a (111)-oriented, epitaxial Ge layer. The fact that this Ge epitaxial layer is fully relaxed is confirmed by the Bragg positions of the Ge (111) peaks, which correspond to the bulk value of the interplanar 
spacing of the $\{111\}$ planes. The full-width-at-half maximum (FWHM) of the $\omega$-rocking curve, measured around the $\mathrm{Ge}(111)$ reflection is $0.3^{0}$.

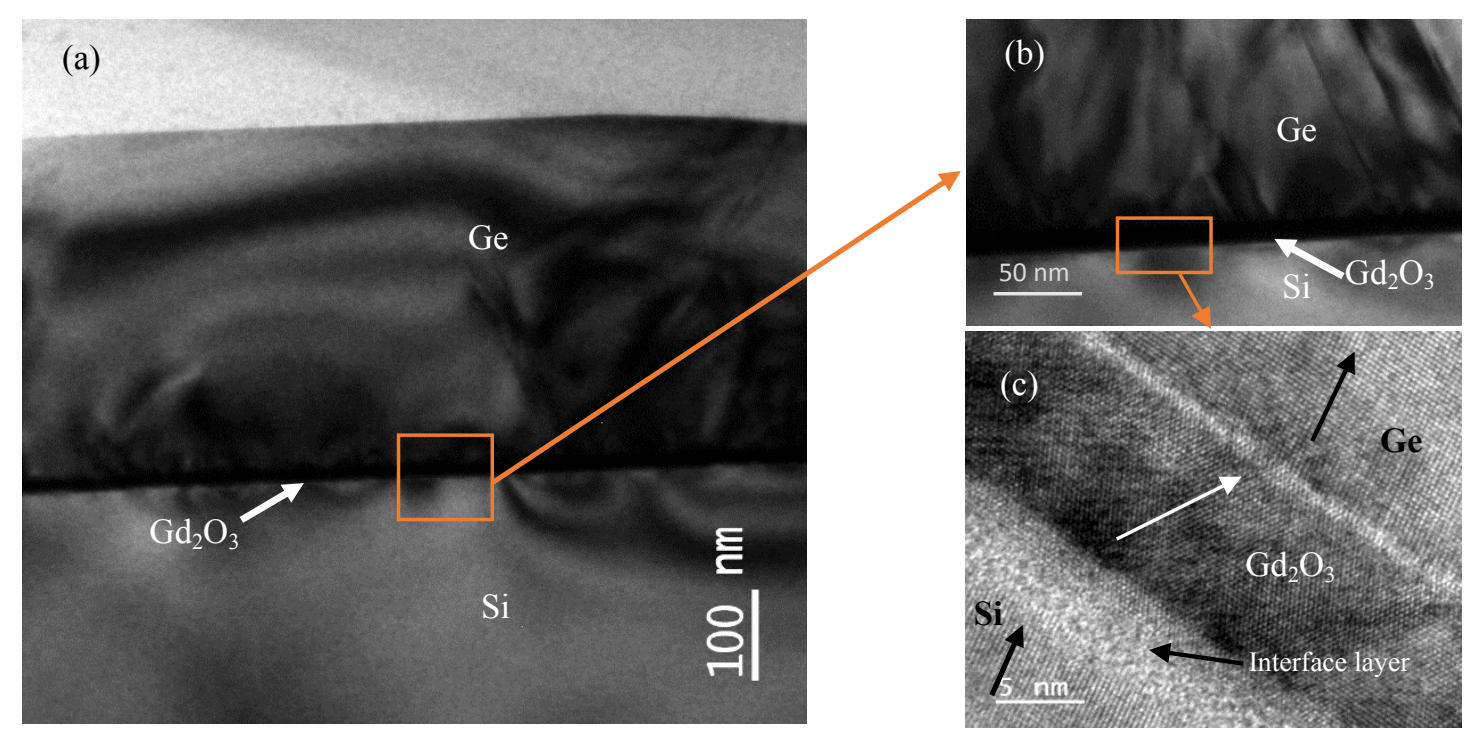

Figure 3. (a) Cross sectional HRTEM image of the $\mathrm{Ge}(111) / \mathrm{Gd} 2 \mathrm{O} 3(111) / \mathrm{Si}(111)$, (b) a close-up image of stacking fault, and (c) a high resolution image of the interface region, showing the stacking orientation of the $\mathrm{Gd} 2 \mathrm{O} 3$ and the Ge epilayers.

XTEM analysis: The TEM image of the grown heterostructure is shown in Figure 3(a). Stacking faults (SF)/twins and threading dislocations are observed near the $\mathrm{Ge}-\mathrm{Gd}_{2} \mathrm{O}_{3}$ interface (Figures 3(a) and 3(b)). The stacking orientation of the Ge and $\mathrm{Gd}_{2} \mathrm{O}_{3}$ epilayers (type-A and type- $\mathrm{B}$, respectively) with respect to the $\mathrm{Si}$ substrate, is seen from the relative orientation of the (1/11) planes in Figure 3(c). An (amorphous) interfacial layer at the $\mathrm{Gd}_{2} \mathrm{O}_{3} / \mathrm{Si}$ interface is also visible in the figure, which forms presumably during the annealing of the substrate at $700{ }^{\circ} \mathrm{C}$.

\subsection{GOI MSM Photodiodes}

A cross-sectional schematic and a microscope-image of the fabricated GOI MSM photodetector is shown in Figure 4(a) and 4(b), respectively. The photodetector has an active area of $100 \mu \mathrm{m} \times 124 \mu \mathrm{m}$, with $4 \mu \mathrm{m}$ finger-width and a pitchlength of $20 \mu \mathrm{m}$. I-V measurements have been recorded in the voltage range of $-2 \mathrm{~V}$ to $+2 \mathrm{~V}$ and $-5 \mathrm{~V}$ to $+5 \mathrm{~V}$. It is observed that for epi-GOI-based MSMs, the dark current is significantly lower than that of MSMs fabricated on Ge wafers (Figure 4(c) and 4(d)). Further, a dark-current reduction of more than a decade is also observed due to annealing of the contacts. The latter effect can possibly be explained by the formation of a n-type region, locally below the metal contacts, in the otherwise (unintentionally) p-type Ge epilayer. This locally-developed n-type regions would form $p$ - $n$ junctions below the metal contacts, thereby providing a MSM device with two back-to-back $p-n$ junctions. Further investigations are underway to ascertain this possibility, as improvement of the passivation layer may also explain reduction in dark current.

At $1550 \mathrm{~nm}$, we observe a rather low photo-response from GOI-based photodetectors. At $1 \mathrm{~V}$ reverse bias, a photocurrent of $\sim 8.6 \mu \mathrm{A}$ is observed, while the dark current is measured to be $475 \mu \mathrm{A}$. At $5 \mathrm{~V}$, the photocurrent increases to $\sim 43.5 \mu \mathrm{A}$ (however, with a back ground dark current of $2.41 \mathrm{~mA}$ ), for a laser power of $24 \mathrm{~mW}$. At the same laser power, bulk-Gebased MSM PDs show a much larger photocurrent $(2.386 \mathrm{~mA})$, with a dark current of only $428 \mu \mathrm{A}$. 


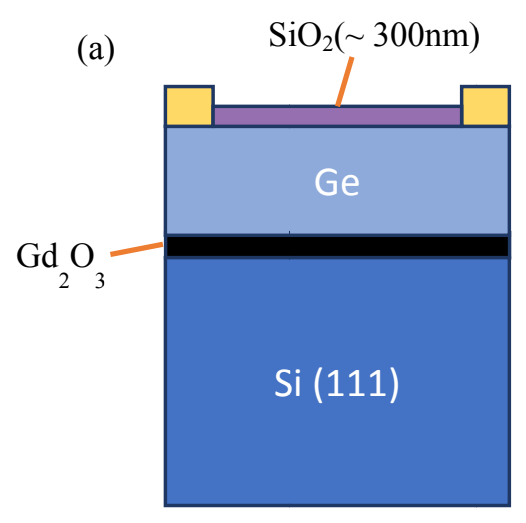

(c) Unannealed

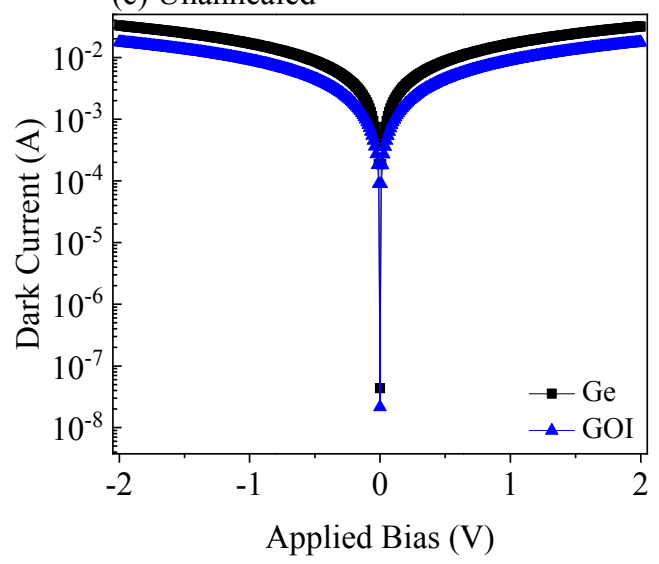

(b)

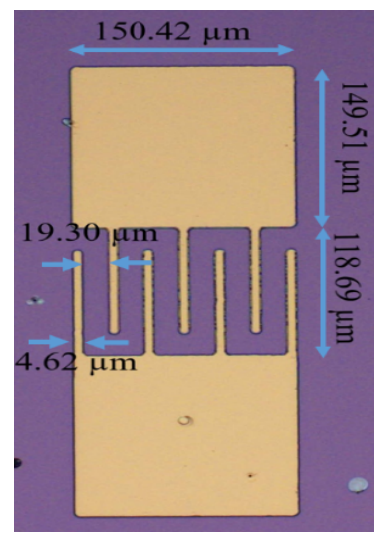

(d) Annealed

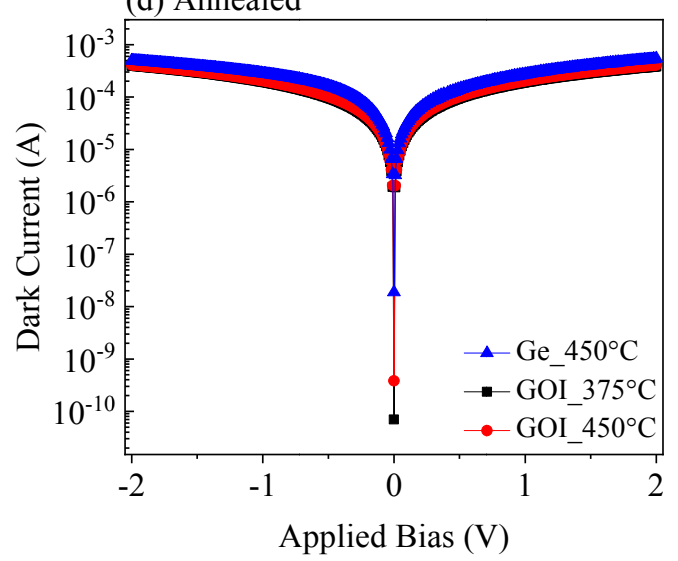

Fig 4. (a) Schematic cross-section and (b) microscope image of the MSM photodetector lay-out. Dark current characteristics of (c) Ge and GOI MSM devices with unannealed contacts (d) and Ge and GOI MSM devices with annealed contacts.

(a) Photoresponse Ge PD

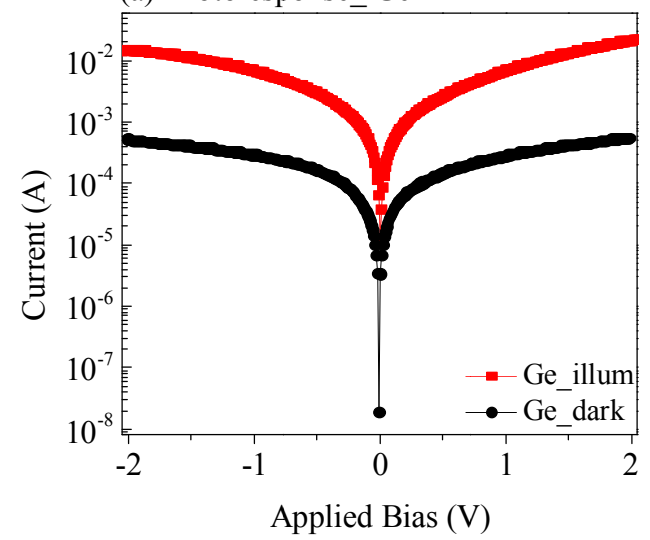

(b) Photoresponse GeOI PD

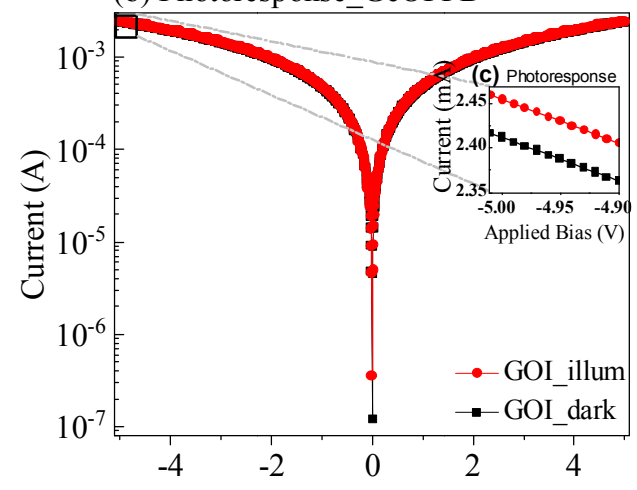

Fig 5. Dark current and photo-response of: (a) Ge MSM photodetector, (b) GOI MSM photodetector. The inset (fig. 4(c)) shows the magnified view of fig. 4(b) near $5 \mathrm{~V}$. The photoresponse from the GOI device is distinctly visible in the inset figure. 
The absorption coefficient of Ge is $460 \mathrm{~cm}^{-1}$ at $1550 \mathrm{~nm}$ wavelength [14] and the above results indicate that epi-Ge layer thickness plays an important role for a good photo-response. The obtained responsivity of GOI MSM devices is $\sim 2$ $\mathrm{mA} / \mathrm{W}$ (at $-5 \mathrm{~V}$ ) with a noise equivalent power (NEP) of $15.32 \mathrm{nW}(\mathrm{Hz})^{-1 / 2}$ [15] (at $1550 \mathrm{~nm}$ ). The low photo-response and high NEP of the GOI photodiode can be attributed to the small thickness of the epi-Ge layers. A thicker epi-Ge layer will both reduce the dark current (due to improved crystal quality) and enhance the photo-response.

\section{SUMMARY}

We reported dark current and photo-response characteristics of all-epitaxial $\mathrm{Ge} / \mathrm{Gd}_{2} \mathrm{O}_{3} / \mathrm{Si}(111)$ germanium-on-insulator MSM photodetectors, and compared the results with those of MSM photodetectors based on bulk-Ge substrates. The hetero-epitaxial structures for the fabrication of the photodetectors have been grown by molecular beam epitaxy. The GOI photodetectors exhibit a dark current of a few hundreds of $\mu \mathrm{A}$, whereas responsivity at $1550 \mathrm{~nm}$ was measured to be

$\sim 2 \mathrm{~mA} / \mathrm{W}($ at $-5 \mathrm{~V})$, with a noise equivalent power (NEP) of $15.32 \mathrm{nW}(\mathrm{Hz})^{-1 / 2}$. Further improvements in the photodetector characteristics may be obtained by increasing the Ge layer thickness.

\section{ACKNOWLEDGEMENT}

This research was funded by the Science and Engineering Research Board, Department of Science and Technology (DST), Government of India. Ravindra Singh Pokharia would also like to thank Kantimay Dasgupta, Aditya Jain, Swarup Deb and Himadri Chakraborty for their assistance during the metallization of the devices. The authors acknowledge the support from the Centre of Excellence in Nanotechnology, Departments of Physics and Electrical Engineering, and Industrial Research and Consulting Centre (IRCC) of IIT Bombay.

\section{REFERENCES}

[1] P. Eng, S. Song and B. Ping, "State-of-the-art photodetectors for optoelectronic integration at telecommunication wavelength," Nanophotonics, vol. 4, no. 3, pp. 277-302, 2015.

[2] V. A. Shah, A. Dobbie, M. Myronov and D. R. Leadley, "High quality relaxed Ge layers grown directly on a Si(001) substrate," Solid-State Electronics, vol. 62, no. 1, pp. 189-194, 2011.

[3] F. Gity, "Development of Germanium/Silicon integration for Near Infrared detection," National University of Ireland, Cork, 2013.

[4] W. Jian, "Fabrication and Characterization of Germanium photodetectors," National Unversity of Singapore, Singapore, 2011.

[5] S. J. Koester, J. D. Schaub, G. Dehlinger and J. O. Chu, "Germanium-on-SOI infrared detectors for integrated photonic applications," IEEE Journal of Selective topics in quantum electronics, vol. 12, no. 6, pp. 1489-1502, 2006.

[6] D. Feng, S. Liao, N. N. Feng, H. Liang, D. Zheng, C. C. Kung, J. Fong, R. Shafiiha, J. Cunningham, A. V. Krishnamoorthy and M. Asgahari, "D. Feng, S. Liao, P. Dong, N.-N. Feng, H. Liang, D. Zheng, C.-C. Kung, J. Fong, R. Shafiiha, High-speed Ge photodetector monolithically integrated with large cross-section silicon-oninsulator waveguide," Applied Physics Letters, vol. 95, p. 261105, 2009. 
[7] L. Vivien, J. Osmond, J. M. Fedeli, D. M. Morini, P. Crozat, J. F. Damlencourt, E. Cassa, Y. Lecunff and S. Lacal, "42 GHz p.i.n germanium photodetector integrated in a silicon-on-insulator waveguide," Optics Express, vol. 17, no. 8, pp. 6252-6257, 2009.

[8] Tracy, C. J. Fejes, P. Theodore, N. D. Maniar, P. Johnson, E. Lamm, A. J. Paler, A. M. Malik, I. J. Ong and Phillip, "Germanium-on-insulator substrates by wafer bonding," Journal of Electronic Materials, vol. 33, pp. 886-892, 2004.

[9] Y. Lin, K. H. Lee, S. Bao, X. Guo, H. Wang, J. Michel and C. S. Tan, "Yiding Lin, Kwang Hong Lee, Shuyu Bao, Xin Guo, Hong Wang, JurgenHigh-efficiency normal-incidence vertical p-i-n photodetectors on a germanium-oninsulator platform," Photonic Research, vol. 5, pp. 702-709, 2017.

[10] J. Wang, A. Laha, A. Fissel, D. Schwendt, R. Dargis, T. Watahiki, R. Shayduk, W. Braun, T. M. Lui and H. J. Osten, "Crystal structure and strain state of molecular beam epitaxial grown $\mathrm{Gd} 2 \mathrm{O} 3$ on $\mathrm{Si}\left(\begin{array}{lll}1 & 1 & 1\end{array}\right)$ substrates: a diffraction study," Semiconductor Science and Technology, vol. 24, no. 4, 2009.

[11] A. Giussani, O. Seifarth, P. Rodenbach, H. J. Müssig, P. Zaumseil, T. Weisemöller, C. Deiter, J. Wollschläger, P. Storck and T. Schroeder, "The influence of lattice oxygen on the initial growth behavior of heteroepitaxial Ge layers on single crystalline PrO2(111)Si(111) support systems," Journal of Applied Physics, vol. 103, p. 084110, 2008.

[12] G. Niu, L. Largeau, G. S. Girons, B. Vilquin, J. Cheng, O. Mauguin and G. Hollinger, "Epitaxial growth of germanium on silicon using a Gd2O3/Si (111) crystalline template," Journal of Vacuum Science and Technology, vol. 28, p. 1187, 2010.

[13] K. R. Khiangte, J. S. Rathore, S. Das, R. S. Pokharia, J. Schmidt, H. J. Osten, A. Laha and S. Mahapatra, "Molecular beam epitaxy and defect structure of Ge (111)/epi-Gd2O3 (111)/Si," Journal of Applied Physics, vol. 124, p. 065704, 2018.

[14] O. Dosunmu, M. K. Emsley, D. D. Cannon, B. Ghyselen, L. C. Kimmerling and M. S. Unlu, "Germanium on double-SOI photodetectors for $1550 \mathrm{~nm}$ operation," in The 16th Annual Meeting of the IEEE Lasers and ElectroOptics Society, Tucson, AZ, USA, 2003.

[15] L. Zeng, L. Tao, C. Tang, B. Zhou, H. Long, Y. Chai, S. P. Lau and Y. H. Tsang, "High-responsivity UV-Vis Photodetector Based on Transferable WS2 Film Deposited by Magnetron Sputtering," Scientific Reports, vol. 6, p. 20343, 2016. 